\title{
LA GÉOGRAPHIE À LA RECHERCHE DES CIVILISATIONS DE HUNTINGTON. ANALYSE DES REPRÉSENTATIONS DU MONDE
}

\author{
Clarisse DIDELON LOISEAU et Yann RICHARD
}

\begin{abstract}
Résumé
La théorie du " choc des civilisations » popularisée dans les années 1990 suite à la publication de l'ouvrage de $\mathrm{S}$. Huntington, connait un large succès politique et médiatique qui ne se dément pas malgré de nombreuses critiques. Dans cet article, nous choisissons de considérer comme possible la représentation du monde en aires de civilisations en conflit entre elles et de tester empiriquement son existence dans un corpus de représentations mentales de l'échelle mondiale produites par des étudiants en s'intéressant particulièrement aux individus appartenant aux civilisations définies comme « islamique » et « occidentale » par S. Huntington.
\end{abstract}

Mots-clés

monde, choc des civilisations, régionalisation, représentations, géographie

\begin{abstract}
The theory of the "clash of civilizations", popularized in the early 1990s following the publication of the book by S. Huntington, knows a wide political and media success which continues unabated despite numerous criticisms. In this article we choose to consider as possible a representation of the world divided in areas of civilisations in conflict among themselves. And we choose to empirically test this hypothesis in a corpus of mental representations of the world produced by the students. We focus attention on individuals in civilisations called 'Islamic' and 'Western'by S. Huntington.
\end{abstract}

Keywords

world, clash of civilizations, regionalization, representations, geography

\section{INTRODUCTION}

La représentation du monde en aires de civilisations, popularisée au $20^{\mathrm{e}}$ siècle par A. Toynbee (1934 à 1961), F. Braudel (1987) et O. Spengler (2000), persiste jusqu'à aujourd'hui (Bruneau, 2010). Elle est explicite dans certaines visions géopolitiques largement diffusées et parfois influentes. On la retrouve de plus en plus mobilisée dans la presse grand public, dans les médias et dans les discours de certains acteurs politiques. J. Cohen rappelle ainsi que même si l'administration Bush ne s'est jamais explicitement réclamée de cette théorie, certaines de ses pratiques témoignent d'un rapport ambigu avec elle (Cohen, 2003). De même, on ne peut que constater la fréquente utilisation de la notion de civilisation par des personnalités politiques variées et nombreuses en France et ailleurs. Le 26 août
2018, Laurent Wauquiez a parlé par exemple de l'immigration de masse comme d'une " menace culturelle pour la civilisation européenne ». Des partis populistes, notamment celui de Viktor Orban, affirment même que l'union politique européenne doit protéger la « civilisation européenne ».

Dans le même esprit, on pense tout naturellement au " Choc des civilisations » de S. Huntington, publié au début des années 1990. La vision du monde proposée dans cette publication a été souvent caricaturée, mais aussi souvent critiquée de façon très convaincante. Toutefois, malgré d'évidentes faiblesses, la théorie du choc des civilisations mérite qu'on s'y intéresse. Premièrement, l'idée de civilisation et d'aires de civilisations est populaire car elle propose une modélisation et une représentation simplifiée du monde. Elle est donc facile à 
utiliser comme levier de mobilisation collective. Deuxièmement, le travail de $\mathrm{S}$. Huntington fait écho à des théories ou idéologies voisines, telles que l'eurasisme qui ressurgit sous sa forme « néo » dans les années 1990 en Russie. Troisièmement, S. Huntington prétend moins fournir un illusoire principe explicatif universel à tous les conflits qu'un paradigme plus efficace que les autres pour observer et interpréter les relations internationales. Quatrièmement, dans les années 1990, il invitait à porter un regard décalé sur le monde post guerre froide, apparemment dominé par les États-Unis. Il prenait le contrepied de l'idée très répandue d'un monde unipolaire. Cinquièmement, S. Huntington constatait le rejet de la domination occidentale dans certaines parties du monde et l'émergence ou la résurgence d'autres acteurs. Enfin, il rappelait aux lecteurs du livre de F. Fukuyama - La fin de l'histoire. Le dernier homme (publié en 1989 aux États-Unis) - que l'histoire n'était pas finie et que le monde n'était pas si transparent et lisse qu'on pensait.

En dépit des critiques fortes qu'elle suscite, nous décidons de prendre cette théorie au sérieux afin d'en tester empiriquement la robustesse. Est-elle partagée ? Nous adoptons une méthode qui repose sur la confrontation avec ce qu'on pourrait appeler les représentations populaires du monde, afin de comparer la vision civilisationnelle proposée par S. Huntington et les représentations du monde de l'homme de la rue, en mettant l'accent sur la relation entre l'aire dite "islamique » et l'aire « occidentale». Pour cela, nous nous appuyons sur une enquête réalisée auprès de plusieurs milliers d'étudiants interviewés dans 18 pays. C'est donc une critique géographique et empirique qui est proposée, en adoptant des approches cartographique et lexicographique. Dans une première partie, nous proposons un rappel des principaux éléments de la théorie du choc des civilisations et nous présentons brièvement les principales critiques qui ont été formulées afin de souligner l'intérêt d'une critique géographique. Dans un deuxième temps, nous présentons les fondements théoriques et méthodologiques de notre approche en présentant notamment l'enquête qui a permis de collecter les informations nécessaires à cette étude. Enfin, nous mettons en œuvre une approche géographique permettant de tester des hypothèses relatives aux représentations du monde que nous avons pu déduire de la théorie de S. Huntington.

\section{LA THÉORIE DU CHOC DES CIVILISA- TIONS. L'APPORT D'UNE APPROCHE DE GÉOGRAPHIE CRITIQUE}

\section{A. Les représentations : pourquoi une comparaison géographique?}

En tant que système d'interprétation, les représentations sociales contribuent à organiser notre relation au monde et aux autres en orientant les conduites et les communications sociales dans divers domaines, entre autres dans la définition des identités sociales (Moscovici, 1961 ; Jodelet, 1984 et 1997 ; Doise, 1990 ; Abric, 1994). Les géopoliticiens leur donnent une place importante au cœur de leurs analyses (Lacoste, 1993). Elles peuvent conditionner les comportements des acteurs et peuvent être à l'origine de conflits (Lacoste, 1993), qu'elles renvoient à des faits objectifs ou non. Ce qui importe dans le cadre de l'analyse géopolitique, c'est que l'attitude des acteurs impliqués est déterminée par la manière dont ils pensent le monde, ou par la manière dont ils se perçoivent eux-mêmes et les autres dans le monde (Boulding, 1958 et 1971 ; Rosière, 2008).

Les auteurs qui se réclament de la géopolitique critique parlent volontiers de geopolitical perceptions ou de geopolitical visions (O'Loughlin et Talbot, 2005). O'Thuatail et al. distinguent quant à eux quatre concepts fondamentaux : la culture géopolitique, les imaginaires géopolitiques, les traditions géopolitiques et les discours géopolitiques (O'Thuatail et al., 2005). Le concept d'imaginaire géopolitique désigne les représentations de l'homme de la rue à propos de son pays. Cela renvoie à ce que Vladimir Kolosov appelle la low geopolitics (ou popular geopolitics), c'est-à-dire « un ensemble de représentations à propos de la place de son pays dans le monde, à propos de l'orientation de sa politique étrangère, de ses alliés potentiels et de ses principaux ennemis » (Kolosov, 2002 et 2003). La low geopolitics se voit dans des «cartes mentales», dans l'usage d'un vocabulaire particulier associé à telles ou telles parties du monde (Henrikson, 1980), ou dans les discours qui expriment l'état d'une opinion publique à propos de la politique étrangère. La high geopolitics se voit dans les discours scientifiques savants sur la politique étrangère et sur les relations internationales, et dans les discours des hommes politiques et des praticiens de la politique étrangère. Ces deux registres doivent être étudiés ensemble (O'Loughlin, Talbot, 2005) car il existe 
Analyse des représentations du Monde

des interactions entre eux (Kolosov 2003 ; Lacoste, 1993). C'est l'idée qu'explore par exemple G. Dijkink dans un article consacré aux relations entre ce qu'il appelle des « codes géopolitiques » et des «représentations populaires » du monde : les élites et les populations participent à la construction des mêmes représentations géopolitiques sans en être toujours conscientes (Dijkink, 1998).

\section{B. Les principales critiques de la théorie du choc}

D'après la théorie de Samuel Huntington, le monde est divisé en aires de civilisations (Figure 1) qui sont les entités les plus larges, dont la délimitation est fondée surtout sur des affinités culturelles. L'influence de l'Occident décline. On observe plutôt un rapport de force entre civilisations. Le monde devient multipolaire, structuré par des formes de coopérations régionales entre des États qui partagent des traits culturels. Les aires de civilisations sont, en général, dominées par des «États phares ». Et c'est à leurs marges que les conflits éclatent.

Cette théorie a été critiquée souvent de façon convaincante. En France par exemple, dès 1995, la revue Culture et Conflits lui a consacré un numéro spécial. D. Bigo (1995) s'interrogeait alors sur la pertinence d'une approche des relations interna- tionales appliquée à des civilisations présentées comme des acteurs à part entière. Il critiquait aussi la place excessive accordée par S. Huntington à l'idée d'affrontement. J. Mueller (1995) critiquait lui l'étonnante souplesse de la pensée de S. Huntington, capable d'adapter son propos et ses idées aux moindres évolutions de la situation internationale au prix de nombreux réaménagements ad hoc, proposant en fin de compte une pensée attrape-tout au prix de raccourcis et d'erreurs. Et W. Jisi (1995) proposait une analyse alternative plus nuancée et non occidentalo-centrée de cette théorie. Selon lui, elle avait le mérite de susciter des questions utiles sur le monde post guerre froide, notamment sur les formes, les lieux et les causes de la conflictualité et sur les facteurs structurants de relations internationales.

D'autres critiques ont été formulées. Cette théorie a un statut ambigu. On ne sait pas s'il s'agit d'une description scientifique de la réalité ou s'il s'agit d'un programme d'action destiné au pouvoir politique. La notion de civilisation est fragile et l'approche qu'en donne Huntington est fixiste car ahistorique. La théorie du choc instaure de l'immuabilité et de l'homogénéité là où il n'y en a pas et relève plus de l'imaginaire que du réel, notamment lorsqu'elle pose l'idée d'un face-à-face entre l'Occident, dont l'existence est sujette à caution (Corm, 2012), et le « Reste », dont le contenu

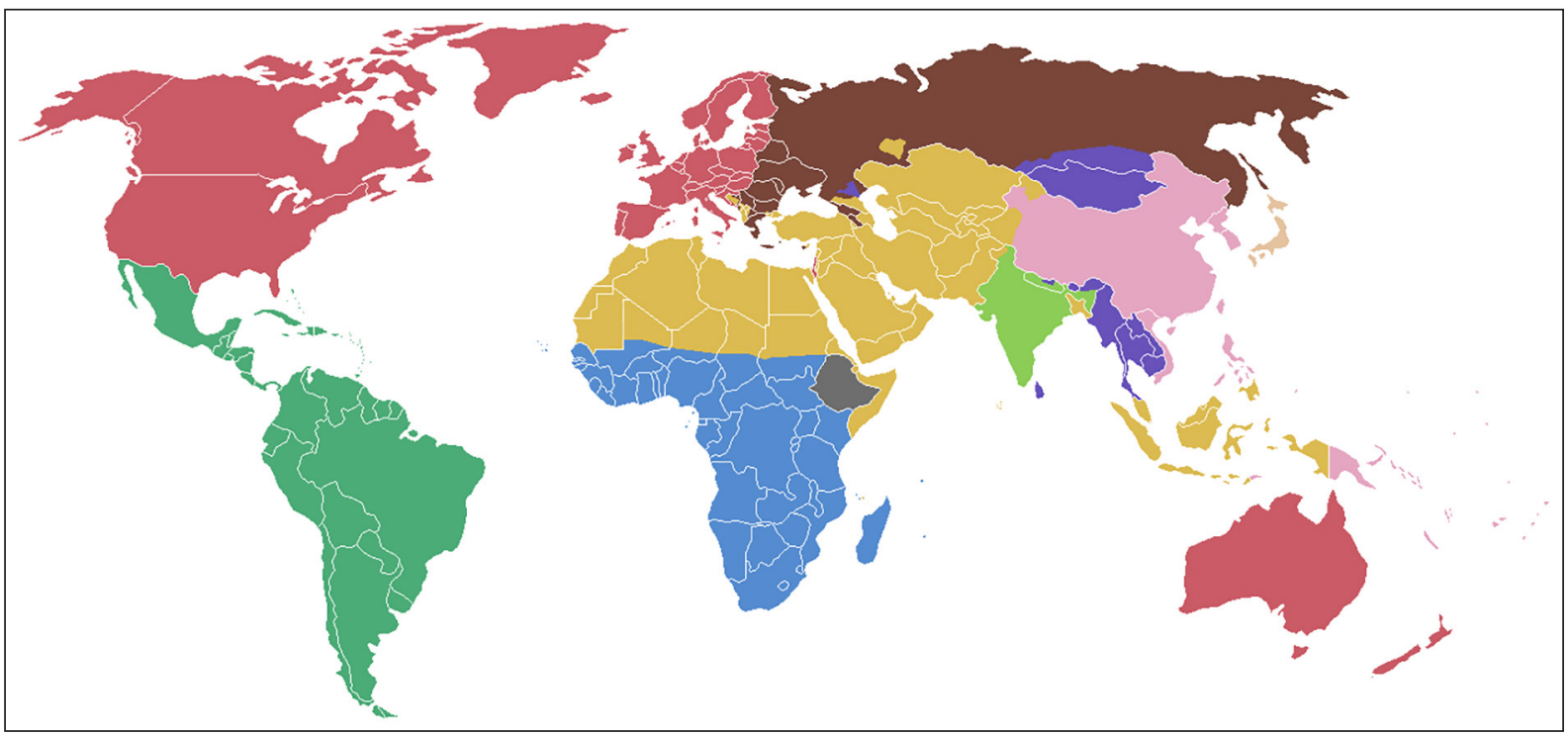

Figure 1. Les civilisations selon Samuel P. Huntington dans Le Choc des civilisations Source : https://fr.wikipedia.org/wiki/Le Choc des Civilisations

Légende : Bordeaux : Civilisation chrétienne occidentale, marron : civilisation orthodoxe, vert bouteille : civilisation d'Amérique latine, ocre : civilisation islamique, vert pomme : civilisation hindouiste, rose : civilisation chinoise, bleu : civilisation africaine, violet : civilisation bouddhiste, chair : civilisation japonaise, gris: pays « isolés » 
n'est pas moins questionnable. Par ailleurs, tout en se plaçant dans le temps long, S. Huntington oublie que la notion de civilisation a elle-même connu bien des évolutions (Claval, 2013), ce qui rend son usage délicat et source d'anachronismes. En réalité, toute identité individuelle ou collective est le produit d'un processus de socialisation, par définition dynamique (Galissot, 1987), en relation avec divers contextes et diverses ressources renvoyant à des registres situés au-delà de la religion et de la culture (Berger et Luckmann, 1996). On peut rappeler à cet égard que les civilisations, si elles existent, ont aussi des bases matérielles et sociales et qu'elles se construisent dans des contextes environnementaux qu'on ne doit pas ignorer (Gourou, 1984 ; Braudel, 1986). Cette théorie est associée à une définition culturaliste et religieuse de l'identité, présentant un risque de dérive essentialiste (Corm, 2012). Les chocs soi-disant culturels masqueraient des conflits qui éclatent pour des motifs souvent plus triviaux (Jisi, 1995). Enfin, les États demeurent les acteurs principaux des relations internationales et le risque d'un axe confucéo-islamique qui s'élèverait contre l'Occident ne renvoie à aucune observation empirique. La théorie des aires de civilisation naturalise l'espace en dessinant un monde divisé en plaques culturelles assimilées hâtivement à des acteurs.

\section{Ce que peut apporter la critique géographique}

En dehors du champ de la géopolitique (Prevelakis, 2013), peu de travaux de géographie proposent une critique ou une déconstruction de cette théorie. Quelques géographes ont néanmoins développé des recherches qui peuvent déboucher sur trois types de critiques. Pour la géopolitique critique, le choc des civilisations est un rideau de fumée qui occulte d'autres choses plus graves : la persistance de la pauvreté, les fortes inégalités de richesse dans le monde, etc. (O'Tuathail et al., 1997). Au-delà de la géopolitique, certains auteurs produisent des recherches qui contredisent ou nuancent la thèse de $\mathrm{S}$. Huntington, sans se référer explicitement à ce dernier. On peut citer les travaux de Christian Grataloup, Vincent Capdepuy et Clarisse Didelon sur les découpages du monde ou sur les limites des continents, soulignant le caractère contingent de ces derniers (Grataloup et Capdebuy, 2013 ; Didelon, 2014). D'autres travaux portent l'attention sur l'intégration régionale dans le monde et, loin des approches culturalistes, développent une interrogation sur le régionalisme Nord/Sud ou sur la construction de macrosystèmes régionaux structurés par de fortes interactions internes. Les limites externes de ces ensembles régionaux multiétatiques sont floues et mouvantes (Beckouche et Richard, 2008 ; Taillard, 2004). Elles ne correspondent ni aux contours des continents conventionnels ni aux aires de civilisations (Beckouche et Grasland, 2008).

Il est tentant de confronter la carte des civilisations avec celle des conflits pour voir s'il existe une correspondance géographique entre elles (Nierop, 2001). Mais une telle confrontation est délicate car elle pose beaucoup de problèmes de méthode. On ne sait pas quels conflits doivent être observés, à quelle échelle il faut les appréhender et comment il faut les cartographier. En réalité, la notion de conflit pose en soi tant de problèmes qu'une confrontation/comparaison cartographique risque de ne pas donner des résultats robustes. C'est une démarche suivie par quelques auteurs (Gurr, 1994 ; Gurr, 2000 ; Russett et al., 2000). Mais dans tous les cas, ces derniers concluent qu'il n'existe aucune preuve empirique d'un lien entre géographie des conflits et géographie des aires de civilisations, si tant est que ces dernières existent.

L'autre approche possible est la confrontation cartographique de la théorie de S. Huntington avec les représentations du monde de l'homme de la rue. Si, à l'instar de V. Kolosov et d'Y. Lacoste, on part de l'hypothèse que les représentations des gouvernants et des praticiens de la géopolitique et celles de l'homme de la rue ne peuvent pas être considérées séparément, on doit admettre que la vision de S. Huntington ne peut pas être vue isolément non plus. Or celui-ci n'appuie jamais son intuition fondamentale sur des enquêtes de terrain. Il se contente de préjuger de ce que pense l'homme de la rue sans l'avoir rencontré. On peut ainsi se demander si, en fin de compte, sa vision n'est pas un placage académique unilatéral sur la carte du monde.

\section{HYPOTHÈSES, MÉTHODE D'ENQUÊTE ET MÉTHODES D'ANALYSE DES REPRÉ- SENTATIONS DU MONDE}

\section{A. Fondement théorique et méthodologique de notre approche}

Un corpus de plusieurs milliers de cartes mentales réalisées à l'échelle mondiale (voir encadré) nous 
Analyse des représentations du Monde

permet d'explorer les représentations du monde de l'échantillon enquêté (des étudiants de niveau licence 3 ) et de déterminer le poids des civilisations de type « Huntington ». Ce corpus, issu du projet EuroBroadMap, est constitué d'un ensemble de découpages du monde en régions, ces régions étant localisées et nommées. L'enquête sur les étudiants menée dans ce projet n'avait pas pour objectif d'analyser les civilisations. Elle a donc été construite pour répondre à une autre problématique $^{1}$. Toutefois, elle a été réalisée dans une perspective et dans des conditions telles qu'elle permet d'analyser les représentations du monde et de faire des comparaisons.

\section{Encadré}

\begin{tabular}{l}
\hline Le projet « EuroBroadMap », financé par la com- \\
mission européenne dans le cadre du 7ème PCRD, \\
regroupait 12 équipes de recherche originaires de \\
11 pays. Dans le cadre de l'un de ses sous-pro- \\
jets, une enquête mondiale sur les représentations \\
du monde a été conduite sur près de onze mille \\
étudiants de niveau « Licence », inscrits dans 6 \\
champs disciplinaires. L'enquête a été réalisée par \\
les membres des équipes partenaires du projet dans \\
43 villes de 18 pays entre juillet 2009 et janvier \\
2010. Elle a permis de récupérer 9360 question- \\
naires entièrement remplis et mobilisables pour \\
l'analyse. L'enquête avait pour objectif d'analyser \\
les représentations de l'Europe dans le monde, \\
mais elle était contrainte par l'instruction émise \\
par la Commission européenne d'être construite \\
de manière non euro-centrique. C'est pour cette \\
raison que les étudiants ont été interrogés sur \\
l'espace mondial, ce qui nous permet de mobiliser \\
ce corpus pour d'autres analyses que celles pré- \\
vues initialement. Il était demandé aux étudiants \\
interrogés de diviser le monde en 2 à 5 régions et \\
de nommer les régions ainsi identifiées. La seule \\
limite est la pertinence de l'échantillon de pays \\
enquêtés, ceux-ci ayant été choisis en fonction des \\
problématiques propres à l'Union européenne au \\
début des années 2010.
\end{tabular}

Les analyses réalisées sur ce corpus ont montré que toute représentation du monde possède deux propriétés que nous pouvons mobiliser dans le cadre d'un questionnement sur le poids des civilisations dans les représentations du Monde. Premièrement les réponses des étudiants montrent une forte tendance au centrage (Didelon-Loiseau, 2013) des cartes qu'ils réalisent sur le pays d'où ils sont originaires et où ils ont été enquêtés. Ce constat est récurrent dans l'analyse des cartes mentales, même à l'échelle mondiale (Saarinen, 1998). Dans cet exercice de régionalisation du monde, cela transparaît dans la numérotation des régions : la région numérotée « 1 », vraisemblablement dessinée et nommée en premier, est celle qui contient le pays où se trouve l'étudiant. Cela signifie que les cartes du monde produites par les étudiants reflètent leur manière de se positionner dans l'espace et leur vision du monde.

Deuxièmement, le fait de dessiner des régions sur une carte du monde conduit les étudiants à construire des aires de coappartenance (Didelon-Loiseau, 2013). En effet, ils placent dans les mêmes régions des espaces qui, selon leurs critères, appartiennent aux mêmes ensembles, comme dans toute démarche classique de régionalisation. Le fait qu'ils se positionnent eux-mêmes sur la carte dans leur démarche de régionalisation conduit à penser qu'ils placent dans la même région que leur propre pays les pays qui leur semblent partager des traits communs, qu'ils soient économiques, culturels... ou civilisationnels. Cela permet d'analyser la composition de la région dans laquelle ils se trouvent, de connaître le nom qu'ils lui donnent et de savoir quels espaces régionaux ils jugent différents de celui auquel ils appartiennent. Compte tenu de ces propriétés, le fait de recourir à des régionalisions du monde pour analyser la place des civilisations dans les représentations, dans une perspective de «low geopolitics », est justifié.

Pourtant, si l'enquête a été menée dans 18 pays, elle ne nous permet pas d'analyser précisément toutes les « civilisations » identifiées par S. Huntington. En effet, nous souhaitions analyser à la fois la manière dont les étudiants se positionnent dans des aires de coappartenance (avec quel(s) autre(s) pays ils s'assemblent et comment ils nomment cet espace régional) et se représentent le reste du Monde (quelles régions ils forment et comment ils les nomment). Notre échantillon, trop petit en termes de pays d'enquête, couvre la majorité des civilisations identifiées par $\mathrm{S}$. Huntington à l'exception des civilisations « bouddhiste» et « japonaise ». De plus, certaines civilisations ne comprennent qu'un pays enquêté : ainsi l'Afrique n'est représentée que par le Cameroun (notre échantillon comprend également le Sénégal, mais S. Huntington le place dans la civilisation islamique) et la civilisation 
latino-américaine uniquement par le Brésil. En revanche, pour la civilisation « islamique » et la civilisation « occidentale », nous avons un nombre significatif de pays. Les enquêtes ont été réalisées en Turquie, en Azerbaïdjan, au Sénégal, en Égypte, en Tunisie, en France, en Belgique, au Portugal, en Suède et en Hongrie (Figure 2). Cet échantillon ne couvre évidemment pas l'ensemble des pays compris dans lesdites « civilisations » : pas de pays d'Arabie ou d'Asie centrale, pas plus que l'Indonésie ; pas d'États-Unis et d'Australie. Pour autant, l'absence de ces pays n'est pas un obstacle majeur. S'il nous faut convenir que les résultats auraient pu être sensiblement différents, notamment en termes de vocabulaire utilisé si nous les avions intégrés, par exemple, nos deux sous-échantillons permettent déjà d'avoir une variété assez grande de points de vue au sein des civilisations. Par ailleurs, si les civilisations sont perçues comme telles, l'absence de l'un ou l'autre des pays mentionnés ne devrait pas avoir d'influence sur le fait qu'elle soit ou non identifiée et nommée.

Les régionalisations du monde des étudiants belges, français, hongrois, portugais et suédois ont été rassemblées dans un sous-échantillon d'étudiants

\section{Civilisations et lieux d'enquête}

Civilisations selon S. Huntington (1990)

Civilisation occidentale

Civilisation islamique

Source des données : Projet EuroBroadMap 2009-11 Réalisation : C. Didelon-Loiseau et Y. Richard, 2017

Figure 2. Pays enquêtés au sein des civilisations identifiées par S. Huntington 
dits « occidentaux », tandis que celles des étudiants azéris, égyptiens, sénégalais, tunisiens et turcs ont été rassemblées dans un sous-échantillon d'étudiants dits " islamiques ». Ce sous-échantillon nous permet de disposer pour l'analyse d'un total de 14490 régions dessinées (Tableau 1). Et on peut d'ores et déjà noter des différences sensibles dans la réalisation de l'exercice par les étudiants. Les étudiants « occidentaux » ont dessiné 7,14 régions en moyenne et les étudiants « islamiques » 5,27, ce qui dénote des différences dans la structuration des découpages du monde (Graphique 1). Tandis que S. Huntington en propose 8. Par ailleurs, dans les deux sous-échantillons, un nombre significatif d'étudiants a décidé de ne dessiner qu'une région. Les étudiants « islamiques » sont plus nombreux à le faire (11\% d'entre eux) que les " occidentaux » $(3,2 \%)$.
Parmi les régions dessinées, 4121 portent un nom qui a été donné par un seul étudiant. En effet, si beaucoup de noms peuvent reprendre des expressions classiques telles qu' « europe », qui fait partie des noms les plus utilisés du corpus, certains étudiants ont utilisé des expressions plus personnelles (par exemple : " countries where I would like to live $\left.{ }^{2} »\right)$, qu'on ne trouve qu'une fois, plus originales que le vocabulaire couramment utilisé (par exemple : «didgeridoo $»^{3}$ ), ou ont proposé des expressions longues et descriptives (par exemple: « developed countries participating to global trade flows »). Ainsi, près d'un tiers des régions dessinées portent un nom unique. Cette proportion varie d'un sous-échantillon à l'autre : les étudiants « islamiques » semblent plus inventifs que les étudiants «occidentaux ». Ces observations ne vont pas dans le sens d'une forte homogénéité du vocabulaire

\begin{tabular}{|l|c|c|}
\hline & $\begin{array}{c}\text { Civilisation } \\
\text { occidentale }\end{array}$ & $\begin{array}{c}\text { Civilisation } \\
\text { islamique }\end{array}$ \\
\hline $\mathrm{Nb}$ d'étudiants & 1358 & 909 \\
\hline $\mathrm{Nd}$ de régions dessinées & 9698 & 4798 \\
\hline $\mathrm{Nb}$ moyen de régions dessinées par étudiants & 7,14 & 5,27 \\
\hline $\mathrm{Nb}$ d'expressions utilisées & 3256 & 2679 \\
\hline $\mathrm{Nb}$ d'expressions uniques & 2193 & 1928 \\
\hline$\%$ d'expressions uniques/Nb de régions & 22,6 & 40,2 \\
\hline
\end{tabular}

Tableau 1. Principales caractéristiques des deux sous-échantillons

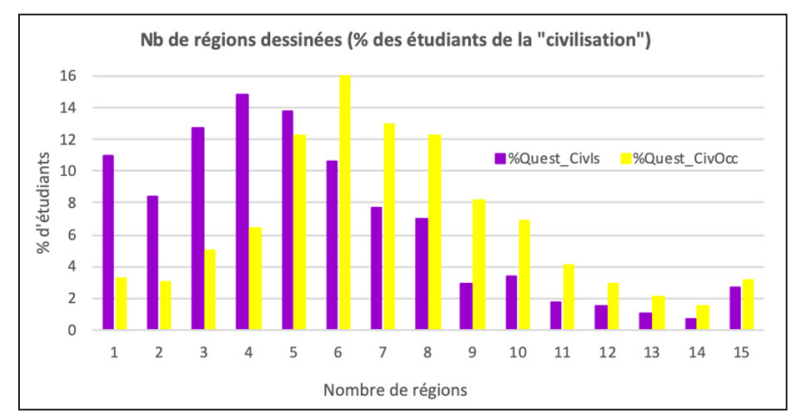

Graphique 1. Nombre de régions dessinées

utilisé, homogénéité que l'on devrait observer si la division du monde en aires de civilisations était dominante.

\section{B. Questionnement et mise en œuvre méthodo- logique}

Pour faire l'analyse, nous adoptons une méthode empirique fondée sur l'hypothèse d'une acceptation de la vision du monde décrite par S. Huntington, c'est-à-dire qu'il y a des civilisations et qu'elles sont en conflit. Que devrions-nous observer dans les représentations du monde des étudiants dans ce cas et comment vérifier cette hypothèse en utilisant le corpus ? Nous disposons dans un SIG de l'ensemble des polygones correspondants aux régions dessinées par les étudiants. À ces polygones est associée une base de données qui les identifie par leur nom et qui permet d'obtenir les informations sur le pays d'origine des étudiants. Ainsi, pour chaque hypothèse nous pourrons faire une série de requêtes permettant d'analyser à la fois la forme des régions et leurs dénominations en fonction des pays d'enquête regroupés en aires de civilisations.

La première hypothèse que nous testons est la suivante : si le monde divisé en aires de civilisations était une vision largement partagée, nous devrions observer, quels que soient les pays considérés et les régions du monde auxquels ils appartiennent, des fortes ressemblances dans les représentations 
du monde des étudiants. Ainsi, il devrait d'abord exister une solide base commune, partagée entre les deux civilisations, et nous devrions observer de fortes corrélations dans l'utilisation du vocabulaire.

La deuxième hypothèse porte sur la conscience des individus d'appartenir à un ensemble. Si la vision du monde divisé en aires de civilisations est vérifiée, elle doit se traduire par des convergences dans les limites des aires régionales, quand bien même ces aires ne portent pas le nom des civilisations. Si cette représentation du monde fait sens, les étudiants originaires de pays localisés dans une aire de civilisation doivent s'identifier spatialement comme «membres » de celle-ci. En toute logique, dans leurs découpages du monde, ils doivent placer le pays dans lequel ils résident dans la même aire géographique que les autres pays faisant partie de cette civilisation. En d'autres termes, pour montrer que l'appartenance commune fait sens pour eux, ils sont censés s'inclure dans le même ensemble régional. En termes de requêtes SIG, pour les pays appartenant à une civilisation donnée (par exemple la civilisation « occidentale »), nous sélectionnons les polygones qui incluent la totalité des pays identifiés par Huntington comme appartenant à cette civilisation (« occidentale »), puis les polygones comprenant au moins un des pays de cette aire. L'objectif est de voir si les pays sont placés dans une même région ou s'ils sont répartis dans des régions différentes et, dans les deux cas, si les noms utilisés correspondent aux noms donnés pas S. Huntington aux aires civilisations. D'autre part, si les civilisations existent, leurs représentants doivent aussi se percevoir mutuellement. Ainsi, pour les résidents d'un pays donné, les pays des autres civilisations doivent être également perçus comme appartenant à un même ensemble géographique identifié. En termes de requêtes SIG, nous procédons de la même manière que précédemment, par aire de civilisation puis par pays.

La troisième hypothèse porte aussi sur le vocabulaire utilisé. Si la représentation du monde est largement structurée par les civilisations, on doit observer l'utilisation d'un vocabulaire ad hoc qui renvoie à la description de ces civilisations, c'est-à-dire des mots tels que "islamique », « occidentale », « orthodoxe », " africaine », dans la dénomination des régions dessinées par les étudiants. En outre, si l'on accepte la logique de l'affrontement et si l'on adhère à la théorie du
" choc », les espaces identifiés comme des civilisations doivent être désignés, au moins en partie, par les étudiants appartenant à l'autre civilisation avec un vocabulaire qui renvoie à l'idée de conflit, de choc et de rivalité.

\section{EXPLORATION EMPIRIQUE. OÙ SONT PASSÉES LES CIVILISATIONS?}

\section{A. Une vision du monde partagée ? Analyse d'un corpus de mots}

L'hypothèse formulée à propos du volet sémantique de l'analyse concerne la cohérence des visions du monde des étudiants et leur compatibilité avec un découpage du monde en aires de civilisations. Ainsi, si la théorie du choc des civilisations domine dans les représentations du monde des étudiants, on doit observer que les étudiants des deux groupes partagent la même vision et cette vision doit correspondre aux aires de civilisations. Pour vérifier cette hypothèse, nous analysons les modalités d'utilisation du vocabulaire utilisé pour nommer les régions dessinées par les étudiants.

Nous avons rapporté la fréquence d'utilisation des expressions utilisées au nombre d'étudiants par pays d'origine et nous avons calculé les corrélations dans l'utilisation du vocabulaire ${ }^{4}$. Le graphique suivant (Figure 3) donne une synthèse des coefficients de corrélation entre les pays. Il permet d'observer des convergences dans la manière de nommer les régions identifiées. Toutefois, un groupe de pays se distingue par une plus grande cohérence dans la manière de nommer les régions, avec des coefficients de corrélations élevés qui traduisent une forte congruence dans l'utilisation du vocabulaire. Ce sont les pays européens de l'échantillon, à l'exception de la Hongrie, mais avec le Sénégal. À l'inverse, les pays de la civilisation « islamique » sont peu corrélés entre eux et certains (Turquie et Tunisie) sont faiblement corrélés avec les pays « occidentaux ». Il faut enfin noter la particularité de l'Égypte et de l'Azerbaïdjan pour lesquels les niveaux de corrélations avec le reste des pays étudiés est très faible. On observe la même chose dans le groupe « occidental» avec la Hongrie. Cela peut s'expliquer par le faible nombre de questionnaires valides recueillis dans ces pays, ou par la forte tendance des étudiants de ces pays à utiliser des noms de régions « uniques ». Ainsi 40,7\% des régions nommées par les étudiants hongrois et $65,3 \%$ de 


\section{Corrélation dans l'utilisation des noms de régions}

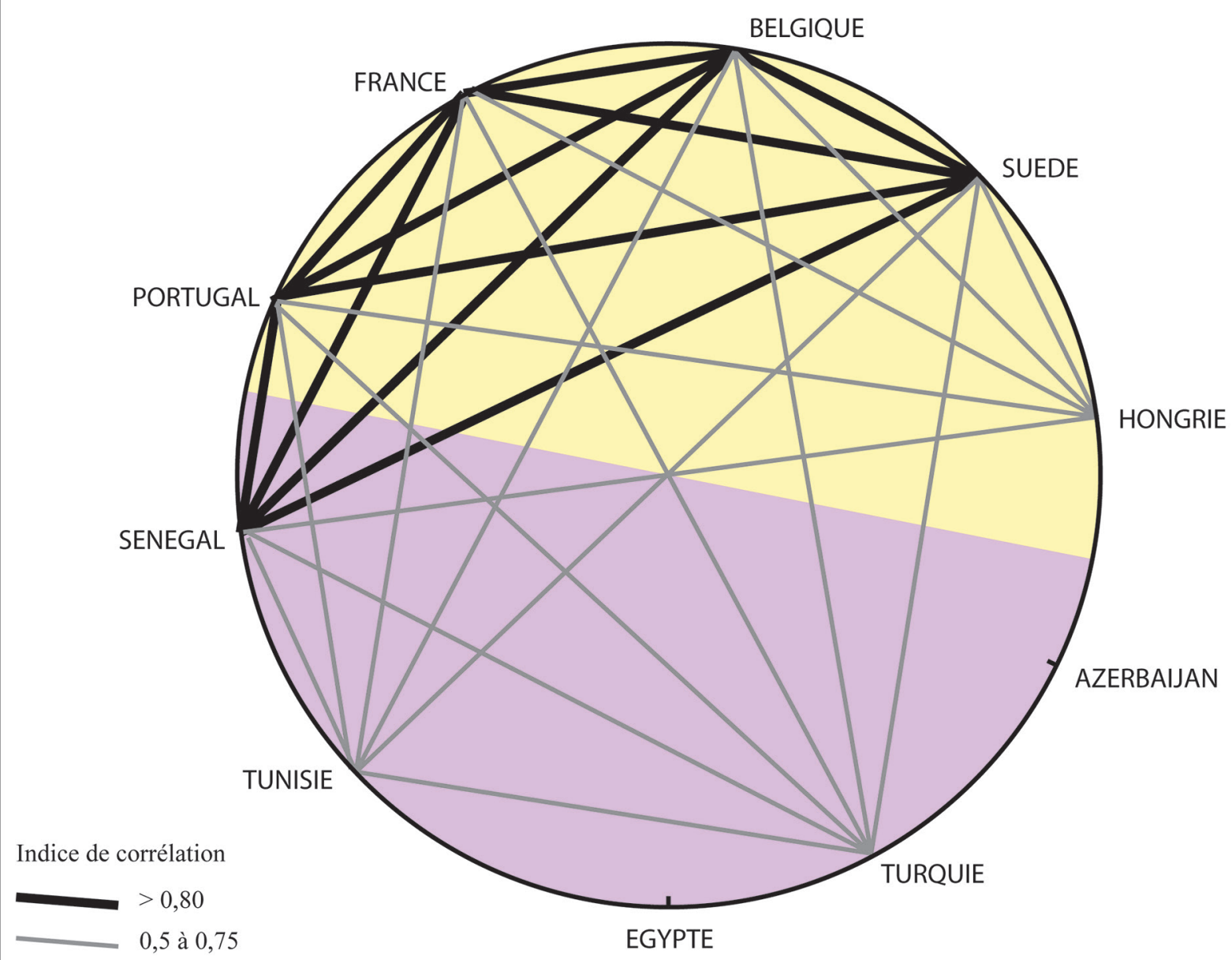

Civilisations selon S. Huntington (1990)

Civilisation occidentale

Source des données : Projet EuroBroadMap 2009-11

Civilisation islamique

Réalisation : C. Didelon-Loiseau et Y. Richard, 2017

Figure 3. Corrélation dans l'utilisation des noms de régions

celles nommées par les étudiants égyptiens ont un nom utilisé une seule fois dans l'échantillon. Toutefois, cette explication n'est que partiellement satisfaisante : $45,6 \%$ des régions nommées par les étudiants turcs ont un nom unique, mais la Turquie et la Belgique ont, par exemple un taux de corrélation de 0.74 dans l'utilisation du vocabulaire tandis que la Hongrie et la Belgique ont un taux de 0.52 seulement.

La concordance du vocabulaire utilisé par les étudiants portugais, français, belges et suédois, témoigne d'une vision commune du monde au sein de l'Europe occidentale héritée des visions du monde élaborées au Moyen Âge, pendant la période des grandes découvertes et pendant les conquêtes coloniales européennes... Dans ce cas, l'influence de l'Europe dans la diffusion d'une certaine représentation du monde (par le biais de la colonisation du Sénégal et de la Tunisie par exemple) ou par l'adoption historique du « modèle » européen (période Atatürk ?) peut expliquer les corrélations avec certains pays. Pour autant, le cas particulier de la Hongrie montre qu'il existe des failles dans la théorie selon laquelle il existerait des visions communes du monde, qu'elles aient un contenu civilisationnel ou pas. Quoiqu'il en soit, nous aboutissons à quelques conclusions : il semble y avoir un socle commun de représentations du monde dans l'espace européen, sauf exceptions; il y a peu de corrélation dans le vocabulaire utilisé entre les deux civilisations et même au sein de la civilisation islamique ; il n'existe pas de vision unanimement partagée du monde en termes sémantiques, ce qui fragilise la théorie du choc des civilisations. 


\section{B. Comment les étudiants découpent-ils le monde?}

Dans un deuxième temps, nous cherchons à retrouver les aires de civilisation identifiées par S. Huntington (Figure 1) dans les cartes mentales des étudiants. Nous avons mis en œuvre des tests cartographiques à partir de la base de données dont nous disposons. Grâce à des requêtes variées dans le SIG utilisé, nous pouvons faire des tests pour savoir dans quel type de structure spatiale se situent les étudiants et dans quel type de structure spatiale ils situent les espaces censés appartenir à l'autre civilisation. Dans cette étape, nous n'observons que la configuration spatiale des régions dessinées pour vérifier si elles correspondent aux configurations spatiales des civilisations de S. Huntington. Ici, un problème méthodologique se pose : les aires de civilisations qu'il identifie sont parfois discontinues. Pour chacune des analyses suivantes, nous utilisons deux requêtes (détaillées ci-dessous) ; pour chacune de ces requêtes nous testons la manière dont les étudiants se situent eux-mêmes sur une carte du monde, c'est-à-dire dans quel type d'espace à l'échelle mondiale ils s'incluent, et la manière dont ils situent les pays de l'autre civilisation.

Avec la première requête, nous cherchons à savoir quelle est la configuration spatiale des régions dessinées incluant la civilisation dans son ensemble (c'est-à-dire qu'il ne peut pas y avoir de limite au sein même de la civilisation). Cela permet de vérifier si les civilisations sont perçues comme un tout. Quel que soit le point de vue (les étudiants d'une civilisation incluant l'autre civilisation ou leur propre civilisation dans une région), les aires incluant le bloc des pays constituant une civilisation sont toujours beaucoup plus larges que les aires définies par S. Huntington (Figure 4). Dans ce cas, les limites dessinées passent rarement à proximité des pays inclus dans les civilisations. Elles sont même plutôt concentrées sur les bords de la carte en projection polaire. Cela indique que, quand les pays appartenant à une même civilisation (par exemple les États-Unis, les pays européens et l'Australie) ne sont pas répartis dans des régions différentes, les étudiants ont dessiné une grande région mondiale. Les deux civilisations sur lesquelles nous concentrons notre analyse ne sont d'ailleurs jamais strictement séparées dans ce cas. Ainsi, visuellement on ne peut pas identifier dans les représentations des étudiants des régions qui correspondraient aux blocs civilisationnels. On voit au contraire que certains étudiants expriment graphiquement une unité entre les civilisations « islamique » et « occidentale», voire entre toutes les civilisations.

Avec la seconde requête, nous cherchons à connaître la forme des régions dessinées incluant au moins l'un des pays de la civilisation (c'està-dire que les pays peuvent être répartis dans des régions différentes) (Figure 5). Cela permet de voir d'éventuelles coupures au sein des civilisations dans les représentations des étudiants. Quel que soit le point de vue, les conclusions sont identiques : les pays constituant les civilisations, loin de faire bloc, sont le plus souvent distribués dans différentes régions du monde. Ainsi, tant pour les étudiants " occidentaux » qu' " islamiques », les États-Unis et le Canada sont souvent séparés des autres pays occidentaux dans une région qui correspond à l'Amérique du Nord ; la Nouvelle Zélande et l'Australie sont situées dans une Océanie ; les pays européens dans une région européenne. De même, les pays de la civilisation " islamique » sont répartis dans au moins deux grandes régions : l'Afrique et l'Asie. Il faut d'ailleurs remarquer que l'espace constitué par les pays du Moyen-Orient et de l'Asie centrale est haché par de nombreuses limites qui séparent les régions dessinées. Il apparaît comme un espace de transition plus que comme le centre d'une hypothétique civilisation « islamique ».

Ainsi, d'un strict point de vue cartographique, l'analyse des représentations du monde des étudiants de l'échantillon contredit l'hypothèse de $\mathrm{S}$. Huntington. Ni les étudiants « occidentaux » ni les étudiants " islamiques » ne s'inscrivent dans un espace qui prendrait la forme d'une civilisation, pas plus qu'ils n'identifient l'autre civilisation. Mais les structures spatiales que nous analysons mettent en jeu des processus de co-inclusion et d'agrégation selon des critères connus ou perçus d'homogénéité qui relèvent d'un certain niveau de complexité. Il est possible que le « choc des civilisations » relève avant tout du discours et de la rhétorique. Si tel est le cas, on doit en trouver la trace dans le vocabulaire utilisé par les étudiants pour nommer les régions, tant pour désigner les civilisations que pour induire une idée d'opposition ou de conflit entre les régions. 


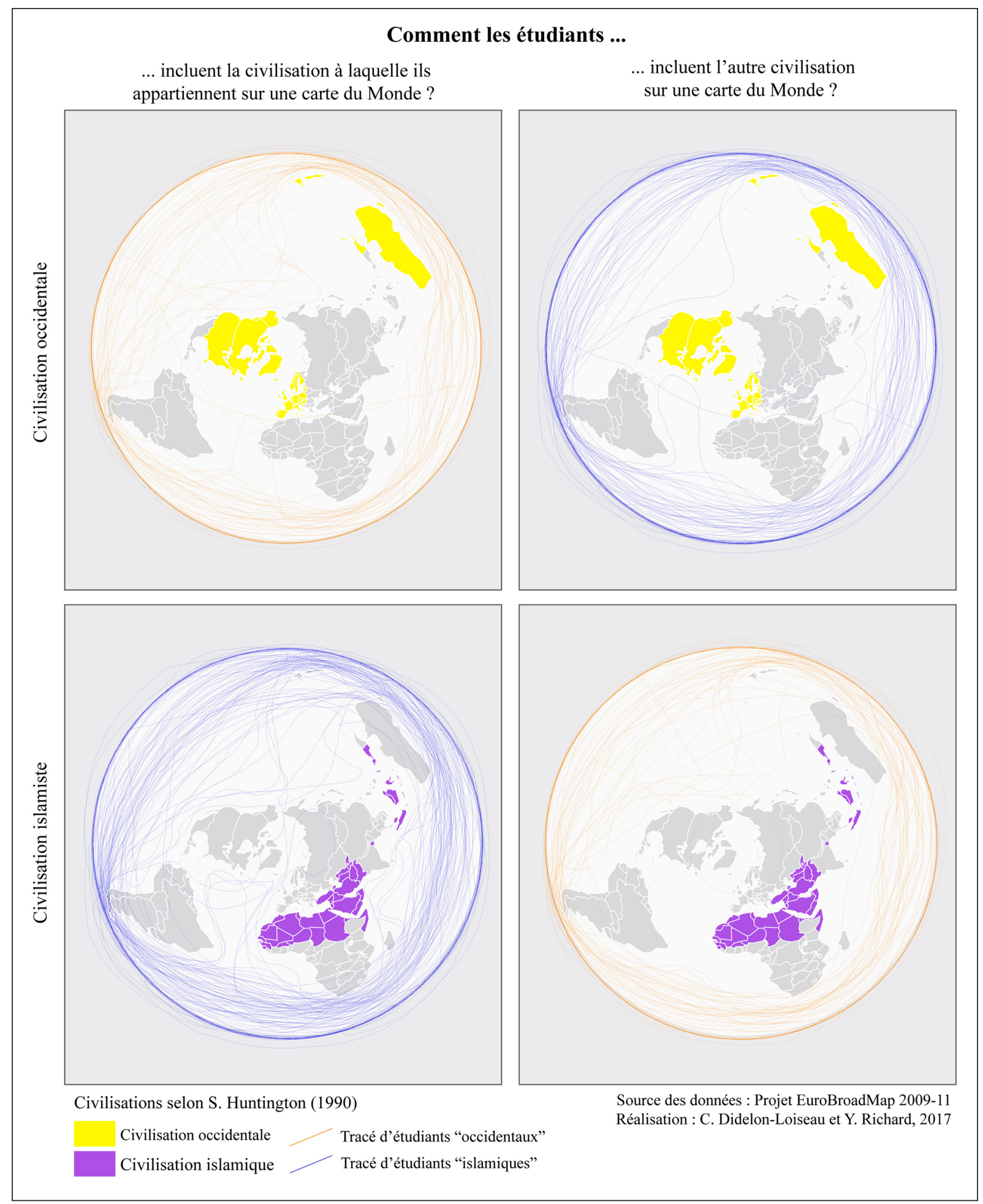

Figure 4. Régions incluant les zones de civilisation

\section{Un choc des civilisations «sémantique»?}

1. Les mots utilisés dans le corpus général des sous-échantillons

Il s'agit d'analyser le vocabulaire associé par les étudiants aux régions dessinées. Nous avons évo- qué précédemment la dispersion du vocabulaire et surtout l'inventivité des étudiants. Notre objectif ici est de savoir à quel point la théorie du choc des civilisations est présente au sein de la population étudiante des civilisations « islamique » et « occidentale ». Dans un premier temps, nous cherchons à savoir si le vocabulaire civilisationnel apparaît 


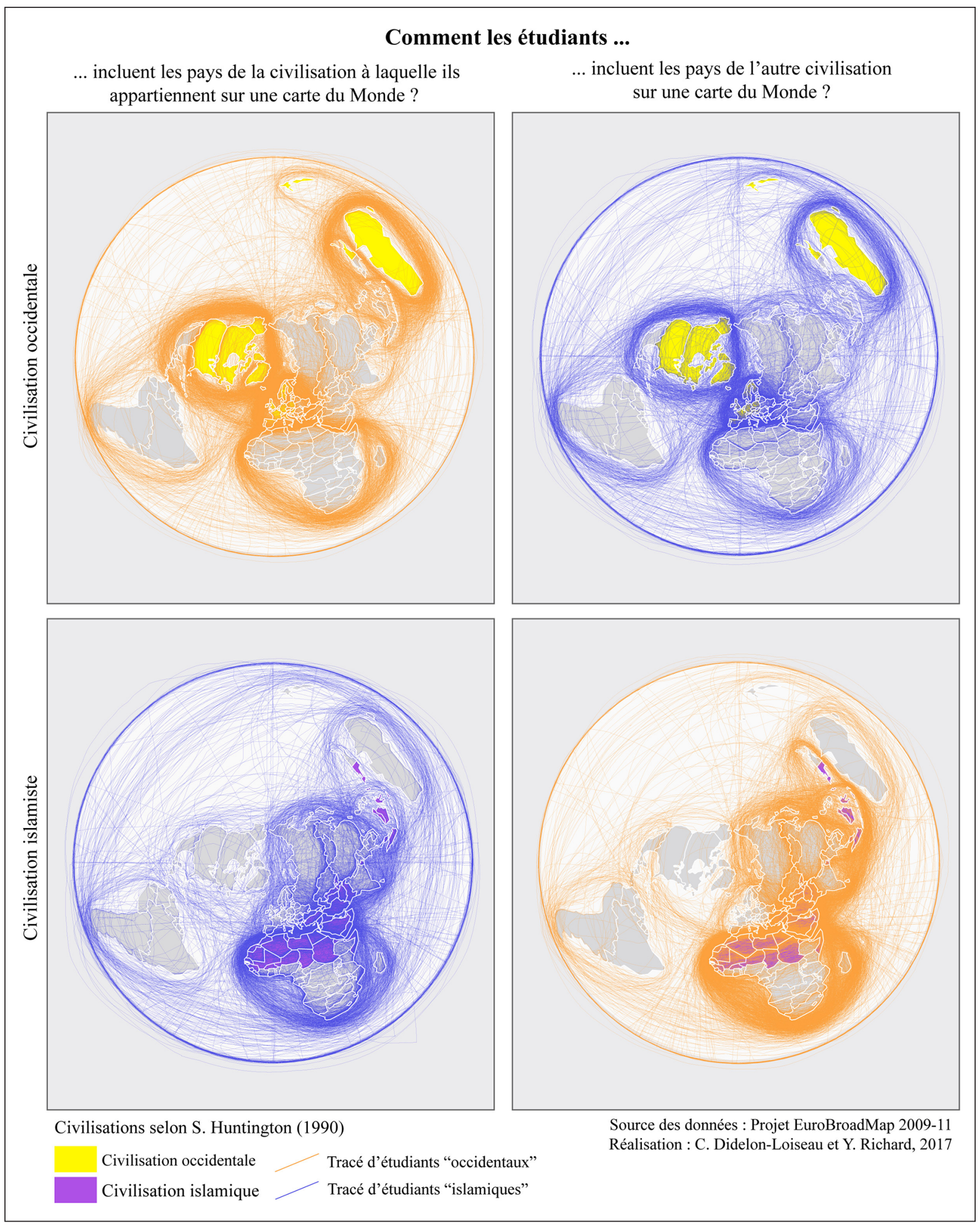

Figure 5. Régions incluant au moins l'un des pays des civilisations

parmi les termes les plus utilisés. Pour chaque aire de civilisation, nous avons sélectionné les noms qui sont utilisés par au moins $3 \%$ des étudiants (Graphique 2). On compte 24 noms de régions différents qui ont servi à nommer 6271 polygones dessinés, soit $43 \%$ du corpus. Il faut noter que, conformément aux observations faites précédemment, les régions citées par les étudiants de la civilisation " occidentale » le sont par une plus grande part d'étudiants. Par exemple, le nom le plus cité par les étudiants des deux civilisations est « europe » : il est cité par 49,6\% des étudiants « occidentaux » et par seulement 20,6\% des étudiants « islamiques ». Ces faibles maximums pour la civilisation « isla- 
Analyse des représentations du Monde

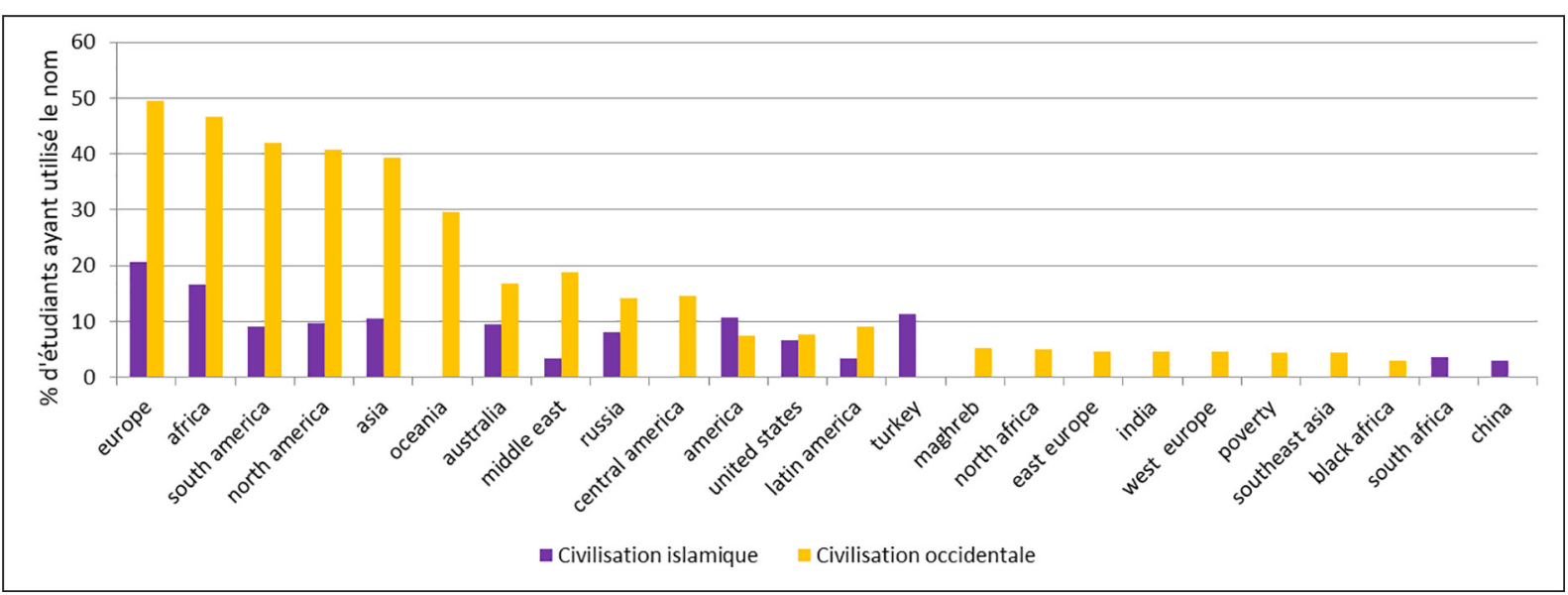

Graphique 2. Noms de régions les plus utilisés (au moins 3\% des étudiants de l'une ou l'autre des civilisations)

mique » sont probablement dus à la forte dispersion du vocabulaire mentionnée plus haut.

Les noms les plus fréquemment utilisés correspondent le plus souvent aux noms traditionnellement donnés aux continents plutôt qu'aux civilisations : « europe », « africa », « south america », «north america $», ~ «$ asia $», ~ « o c e a n i a ~ »$. On trouve ensuite des noms de régions souvent utilisés dans un contexte géographique ou géopolitique tels que « middle east » ou « west europe », " east europe $»$. Ou encore des noms de pays : «australia », « russia », «turkey » ou « india ». Aucun terme, parmi les plus cités ne correspond directement aux noms des civilisations : les termes occident ou occidental («west » ou «western ») ou islamique («islamic ») n’apparaissent pas en tant que tels. Le terme «west » figure bien sur le graphique, mais seulement comme une sous-région de l'espace européen : Europe de l'ouest ou Europe occidentale. Quant aux termes « maghreb» ou « middle east », ils renvoient à un vocabulaire classique et neutre de désignation des parties du monde en géographie ou en géopolitique et, d'un point de vue spatial, ils ne correspondent qu'à des portions de la civilisation « islamique » identifiée par S. Huntington.

\section{2. À la recherche des civilisations}

Dans son ensemble, le vocabulaire utilisé par les étudiants ne contient pas de termes fréquemment utilisés qui feraient référence aux civilisations. Nous avons alors cherché à mesurer la part des noms donnés aux régions qui font référence aux civilisations telles que les nomme ou les décrit S. Huntington. Nous avons, à chaque fois, analysé le point de vue des étudiants sur leur propre civilisation et sur l'autre civilisation.
Pour ce faire, nous avons cherché dans le corpus des noms de région le nombre d'occurrences des chaînes de caractère suivantes : « islam », « muslim » et 《 arab $\rangle^{5}$ pour décrire la civilisation « islamique »; « west », « occid» et « christ » (et ses déclinaisons possibles, 《 catho », 《 protest ») pour décrire la civilisation « occidentale ». Le Tableau 2 montre les occurrences observées dans l'échantillon. On peut voir qu'elles sont peu nombreuses. Pour désigner la civilisation « occidentale », le vocabulaire religieux n'est pratiquement jamais mobilisé ; de plus, le vocabulaire huntingtonien est rarement utilisé dans plus de $1 \%$ des cas pour nommer les régions. Pour désigner les « islamiques », les dénominations de S. Huntington ne sont pas davantage utilisées, sauf quand ce sont les « islamiques » qui nomment leur propre région. On atteint alors 1,5\% des noms quand les régions incluent au moins l'un des pays de la civilisation et même 5,2\% des noms quand elles comprennent l'aire de civilisation « islamique » identifiée par S. Huntington (Tableau 2). La chaîne de caractère « arab » est la plus utilisée à la fois par les « islamiques » et les « occidentaux », pour qualifier certains lieux géographiques bien identifiés (péninsule arabique, Arabie Saoudite) ou des espaces aux limites plus floues (monde arabe, pays arabes).

Quand on analyse les noms des régions qui incluent entièrement les civilisations cartographiées par $\mathrm{S}$. Huntington, les termes utilisés pour les nommer reprennent rarement son propre vocabulaire. Au contraire, que l'observateur soit « islamique » ou « occidental », ce sont les mots « world» $65 \%$ des occurrences pour les islamiques et $40 \%$ pour les occidentaux), « earth » $(15 \%$ d'occurrences pour les occidentaux seulement) qui reviennent le plus souvent (ce qui confirme les analyses faites 


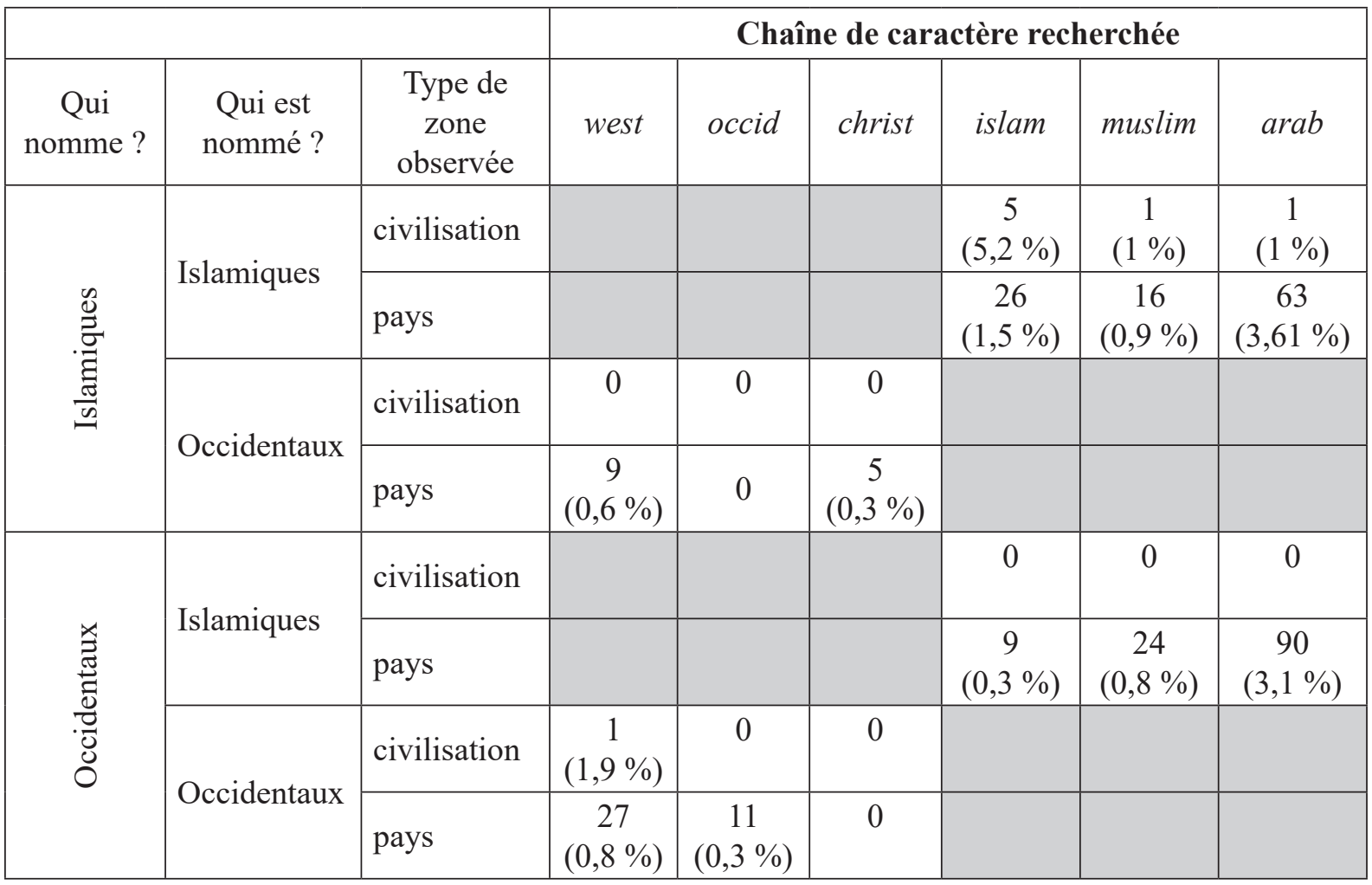

Tableau 2. Occurrence des termes de référence des civilisations

précédemment à propos du tracé des limites des régions dessinées), utilisé pour désigner le monde dans son ensemble, à quelques exceptions près. Au-delà du mot désignant le monde, les expressions d'échelle mondiale, telles que «world», mais aussi les expressions telles que "love different people different opinions wishes love \& respect get them all together », «peace », « we are brothers », ou encore "world people should not be divided according to the places they live race \& religion $»$, représentent $72,2 \%$ des occurrences des noms de régions donnés par les étudiants « islamiques ». À deux reprises seulement, le mot « world » est utilisé pour désigner une région particulière : un «muslim world » et «near future turk islam world ». Une seule fois, il est également utilisé pour désigner le monde moins un pays : « united world except israel ». Le Tableau 2 , montre que la chaîne de caractère « west » n'est utilisée qu'une fois, le monde étant de fait sur la carte en question divisé entre un ouest et un est qui correspondent plutôt aux côtés gauche et droit de la carte en projection polaire. D'ailleurs, on voit sur d'autres cartes des partitions en nord et sud.

\section{Un monde conflictuel?}

Dans le lexique mobilisé par les étudiants, nous ne retrouvons donc pas trace d'un vocabulaire renvoyant aux civilisations. Nous nous sommes donc finalement demandé si, dans les termes utilisés, nous pouvions retrouver un vocabulaire renvoyant à l'idée de conflit, liée à l'idée de « choc des civilisations ». Pour identifier le vocabulaire « conflictuel», nous avons procédé en trois temps. Chacun de nous a dressé une liste de termes qu'il s'attendait spontanément à trouver dans le corpus des mots utilisés par les étudiants compte tenu de la lecture de S. Huntington. Nous avions, par exemple, retenu des termes comme « intolérant», « exploitation », « intégrisme », « arrogant », etc. Nous avons ensuite cherché et localisé ce corpus, une fois traduit en anglais, dans le vocabulaire ${ }^{6}$. D'autre part, nous avons séparément examiné la liste des 3237 expressions utilisées pour distinguer celles qui semblaient avoir une dimension conflictuelle, comme les expressions «place where oppressed \& sweated people live » et «area of religious fanaticism ». À ce stade, une grande partie du vocabulaire lié au conflit était identifiée de manière concordante par les deux auteurs de l'article, mais il existait quelques divergences (77 au total) sur lesquelles nous avons statué ensemble.

En fin de compte, nous avons identifié seulement 496 occurrences d'expressions ayant une dimension conflictuelle, ce qui représente $5,27 \%$ des 
Analyse des représentations du Monde

expressions utilisées par les étudiants. Ces expressions peuvent désigner l'autre civilisation mais aussi sa propre civilisation. Le poids du vocabulaire lié à l'idée d'un choc entre les deux aires de civilisations est donc marginal. Le tableau ci-dessous rend compte de la répartition des occurrences. On y voit se dessiner une différence assez nette entre les étudiants « islamiques » et les étudiants « occidentaux » (Tableau 3). Les premiers semblent avoir plus fréquemment recours à un vocabulaire à connotation conflictuelle, que ce soit pour désigner les « occidentaux » ou pour se désigner eux-mêmes $(9$ à $10 \%$ du corpus utilisé). Les seconds ont presque moitié moins tendance à utiliser un vocabulaire conflictuel pour désigner les « islamiques » et encore moins pour se désigner eux-mêmes.

Par ailleurs, le vocabulaire utilisé appartient à des registres différents. Comme l'illustre la Figure 6, le mot le plus fréquemment utilisé par les étudiants « islamiques » pour désigner les pays appartenant à l'aire occidentale est « imperialist » (22 occurrences), dans des expressions aussi diverses que "west imperialist \& capitalist », qui semble relever $\mathrm{du}$ registre du choc des civilisations selon les critères de $\mathrm{S}$. Hungtington, mais aussi plus simplement «imperialist countries ». On retrouve dans ce corpus des termes qui dépeignent les pays occidentaux comme impérialistes, exploiteurs, colonialistes, racistes, égoïstes et autoritaires. On est donc bien dans un vocabulaire qui établit une relation entre les deux régions, une relation fondée sur un rapport de domination largement hérité de la colonisation. Cela se retrouve d'ailleurs dans le vocabulaire utilisé par les islamiques pour se désigner eux-mêmes puisque l'on retrouve parmi les expressions utilisées des termes comme « exploited ( 37 occurrences), « oppressed » ou « underdevelopped 》 utilisé explicitement dans un contexte relationnel comme dans l'expression « underdeveloped countries by their imperials».

Le vocabulaire utilisé par les « occidentaux » pour nommer les pays appartenant à la civilisation « islamique » a une dimension moins relationnelle et plus descriptive (Figure 7). Il sert à décrire une situation de conflit et de guerre au sein de l'aire islamique, dans laquelle il est dangereux de se rendre, comme l'indiquent les expressions « conflict area », " countries in war" ou encore "dangerous area to live ». Le terme «war» est ainsi présent dans 41 des 139 expressions utilisées par les occidentaux, soit près de $30 \%$ mais jamais dans une expression de type relationnelle. Ces conflits et guerres sont parfois identifiés comme des conflits religieux. La dimension religieuse est relativement présente d'ailleurs dans l'utilisation d'un vocabulaire péjoratif tel que « fanaticism», mais, encore une fois, moins dans un sens relationnel que dans un sens descriptif comme dans l'expression « area of religious fanaticism ». Dans les rares expressions à connotation conflictuelle utilisées par les « occidentaux » pour

\begin{tabular}{|l|c|c|c|c|c|}
\hline & Isl=>Occ & Isl=>Isl & Occ=>Isl & Occ=>Occ & Total \\
\hline Termes « conflictuels » & 148 & 162 & 139 & 47 & 496 \\
\hline Nombre de termes utilisés & 1496 & 1741 & 2943 & 3237 & 9417 \\
\hline$\%$ de termes Y\&C & 9,89 & 9,30 & 4,72 & 1,45 & 5,27 \\
\hline
\end{tabular}

Tableau 3. Fréquence d'utilisation d'un vocabulaire à connotation conflictuelle

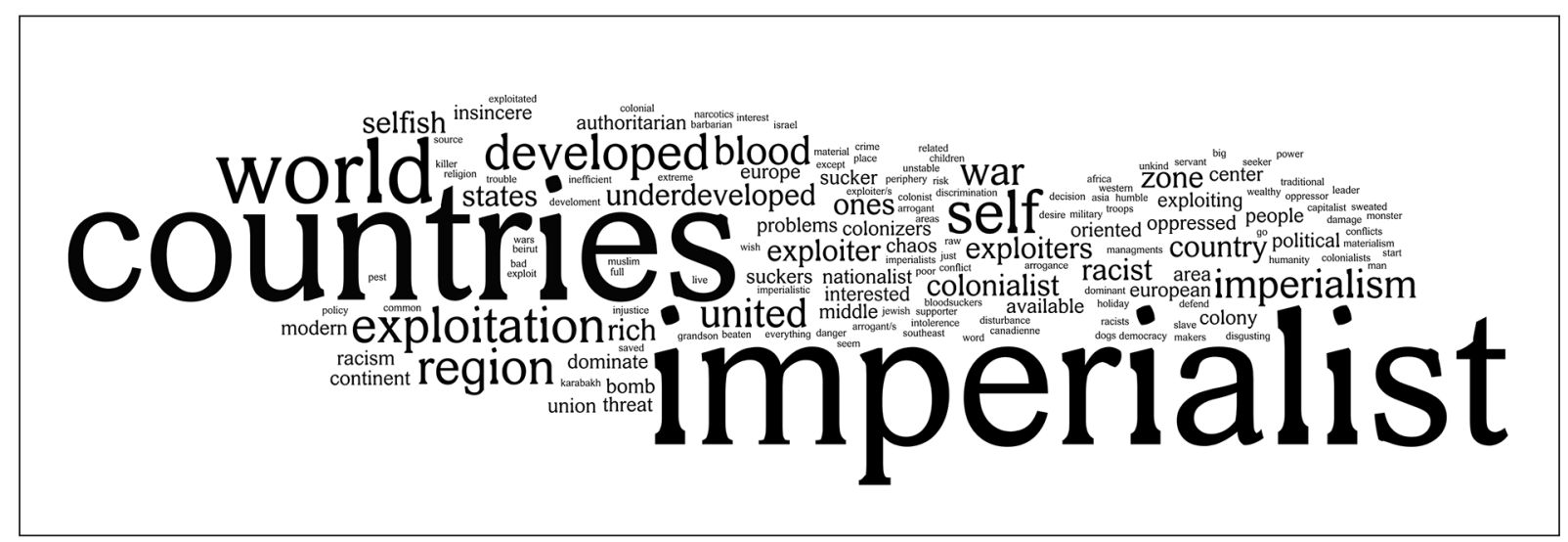

Figure 6. Le vocabulaire à dimension conflictuelle utilisé par les islamiques pour désigner les occidentaux 


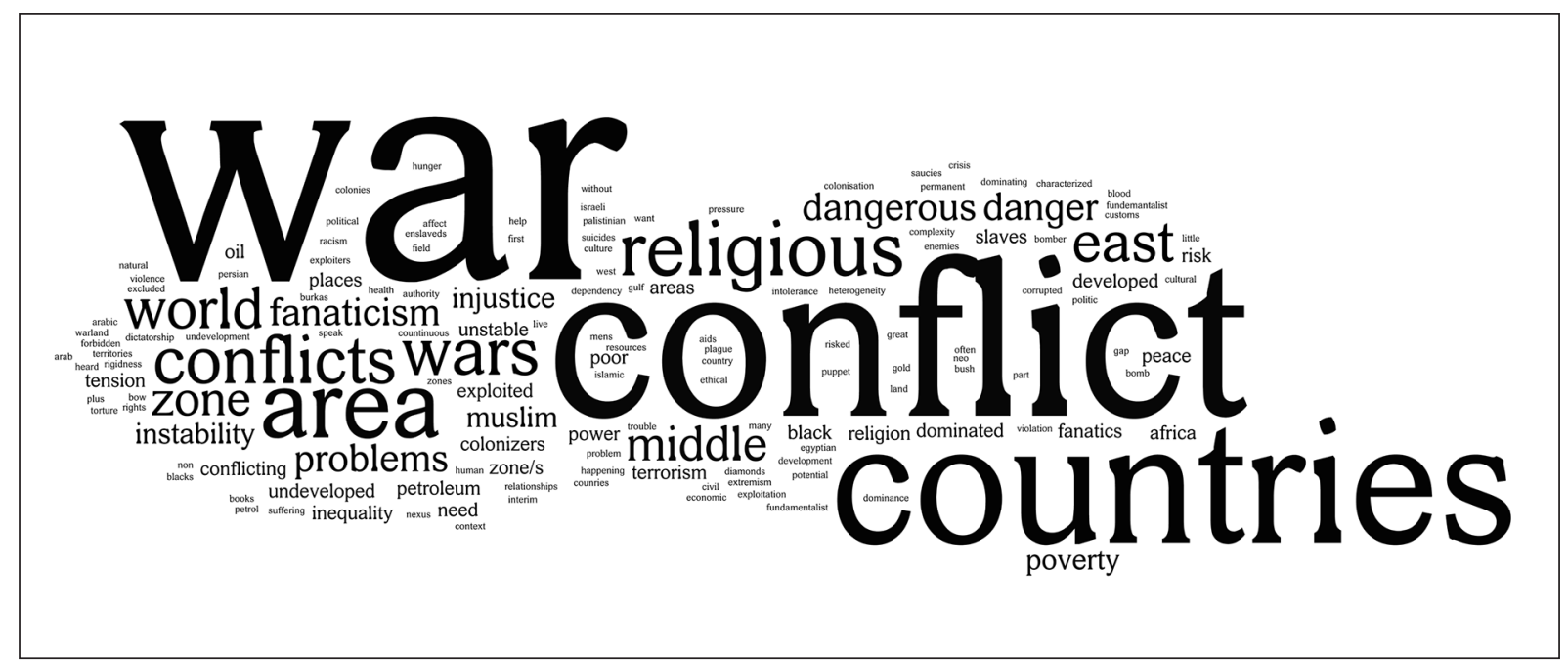

Figure 7. Le vocabulaire à dimension conflictuelle utilisé par les occidentaux pour désigner les islamistes

se décrire eux-mêmes, on retrouve en revanche des expressions relationnelles. Ils font alors référence à la position de domination des pays dans lesquels ils vivent, comme dans l'expression « west first world exploiters ».

Ainsi, la dimension conflictuelle est bien présente dans le vocabulaire utilisé par les étudiants pour nommer les régions, mais le poids de ce vocabulaire dans le corpus est marginal. Cela confirme la fragilité de la vision de S. Huntington car il n'y a pas de symétrie ou de convergence dans les manières dont les « islamiques » et les « occidentaux » se perçoivent mutuellement et se perçoivent eux-mêmes.

\section{CONCLUSION}

La vision civilisationnelle du monde proposée par Samuel Huntington a été souvent critiquée dans les années 1990 et dans les années 2000. Les résultats obtenus dans cet article s'inscrivent donc dans une tradition déjà longue, de discussion de l'hypothèse du choc des civilisations. Ils permettent toutefois d'aller encore plus loin en soulignant une faiblesse du travail de S. Huntington qui n'est pas explorée dans les nombreuses critiques qui lui ont déjà été adressées. Il a proposé une vision du monde présentée comme un fait établi, comme un "déjà là » positivement attesté. Or plusieurs de nos conclusions permettent de le contredire. (i) Le découpage du monde en aires est en général classiquement continental ; les aires de civilisations dessinées par S. Huntington n'apparaissent pas dans l'enquête. (ii) Parmi les pays « islamiques », il n'existe pas de convergences dans le vocabulaire utilisé par les étudiants pour désigner les régions qu'ils dessinent sur les cartes du monde. (iii) Les noms les plus souvent utilisés désignent eux-mêmes des continents et pas des civilisations. (iv) Enfin, le poids relatif du vocabulaire à connotation conflictuelle utilisé pour désigner les régions dessinées est marginal. En bref, rien n'atteste que les étudiants interrogés se retrouvent dans la vision d'un monde divisé en aires de civilisations et encore moins dans l'idée d'un choc entre celles-ci. En d'autres termes, la low geopolitics ne croise pas la route de la high geopolitics.

Le travail empirique et cartographique proposé ici présente néanmoins quelques limites. S. Huntington est un auteur américain, or nous n'avons pas pu mener notre enquête aux États-Unis. On peut faire 1'hypothèse que des étudiants américains proposeraient des visions du monde convergentes avec l'idée de choc des civilisations. Cela reste à vérifier. Par ailleurs, l'échantillon de population n'est composé que par des étudiants. Il s'agit d'une population très particulière et on peut penser que leurs représentations du monde ne ressemblent pas à celles qu'on pourrait observer dans d'autres catégories de population. Enfin, on peut raisonnablement penser que les représentations mentales évoluent dans le temps, notamment à l'échelle mondiale. Le conflit en Syrie, les attentats islamiques en Europe et aux États-Unis... et la réapparition régulière du thème des civilisations dans les médias peuvent contribuer à diffuser la vision de $\mathrm{S}$. Huntington dans l'opinion publique de certains pays.

\section{NOTES}

${ }^{1}$ La question posée était : « Sur la carte suivante, dessinez vos propres régions du monde (15 au maximum), donnez 
Analyse des représentations du Monde

un numéro et un non à chacune de ces zones.

${ }^{2}$ Le corpus que nous analysons a été traduit en anglais depuis les différentes langues nationales dans lesquelles l'enquête a été réalisée.

${ }^{3}$ Instrument de musique à vent d'origine aborigène.

${ }^{4}$ Les corrélations ont été calculées sur l'ensemble du corpus, c'est-à-dire les 4838 termes utilisés au moins une fois. Toutefois, comme il existe un grand nombre d'expressions utilisées seulement une fois (4 121), nous avons vérifié la stabilité de la mesure en calculant les corrélations sur les 226 expressions utilisées au moins 5 fois, puis sur les 123 expressions utilisées au moins 10 fois. Les coefficients de corrélations restent particulièrement stables.

${ }^{5}$ La chaîne de caractère fait référence à l'Arabe comme langue ou aux Arabes, c'est-à-dire les personnes de langue ou de culture arabe. Elle exclut de fait des populations musulmanes non arabophones (Indonésie). Toutefois nous avons choisi d'analyser la présence de ce terme dans le corpus car nous supposions la possibilité de l'existence d'une confusion, pour « l'homme de la rue » entre le fait de parler arabe et d'être musulman. ${ }^{6}$ Nous avons procédé par recherche de chaîne de caractères.

\section{BIBLIOGRAPHIE}

Abric, J.-C. (1994). Pratiques sociales et représentations, Paris, PUF.

Alker Jr, H.R. (1995). Pour qui sont ces civilisations? Cultures \& Conflits, 19-20, parties 1 et 2. URL : http://journals.openedition.org/conflits/882 ; DOI : 10.4000/conflits.882

Beckouche, P., Grasland (et alii) (2008). Europe in the World. Territorial Visions and Evidence. Paris, Luxembourg, CNRS, Diact, Université Paris 7 Denis Diderot, Université Paris 1 Panthéon Sorbonne, Espon Programme.

Beckouche, P. \& Richard, Y. (2008). Atlas d'une nouvelle Europe. L'UE et ses voisins : vers une région mondiale? Paris, Autrement.

Berger, P. \& Luckmann, T. (1997). La construction sociale de la réalité. Paris, Armand Colin.

Bigo, D. (1995). Grands Débats dans un Petit Monde. Cultures \& Conflits, 19-20, parties 1, 2 et 3. URL : http://journals.openedition.org/conflits/872 ; DOI : 10.4000/conflits.872

Boulding, K.E. (1958). National Images and International Systems. Conflict Resolution, 3(2), 120-131.

Boulding, K.E. (1971). National images and international systems. In Hanrieder W.F. (ed.), Comparative foreign policy. Theoretical essays, New York, McKay, 90-107.

Braudel, F. (1979). Civilisation matérielle, économie et capitalisme XVE-XVIIIe siècle. Paris, Armand Colin.

Braudel, F. (1987). Grammaire des civilisations. Paris, Arthaud.
Bruneau, M. (2010). Civilisation(s) : pertinence ou résilience d'un terme ou d'un concept en géographie? Annales de géographie, 674, 315-337.

Claval, P. (2013). L'idée de civilisation dans la pensée contemporaine. L'apport de la géographie et de l'histoire. Anatoli, 4, 57-76.

Cohen, J. (2003). Samuel Huntington dans l'univers stratégique américain. Mouvements, 30, 21-30.

Corm, G. (2012). L'Europe et le mythe de l'Occident. Paris, La découverte.

Corm, G. (2012). Pour une lecture profane des conflits. Paris, La découverte.

Didelon-Loiseau C. (2013). Le Monde comme territoire, pour une approche renouvelée du Monde en géographie. Habilitation à diriger des recherches. Université de Rouen.

Didelon, C. (2014). Régionalisations mentales du monde. In A. Gana et Y. Richard (ed.), La régionalisation du monde, Paris, Tunis, Karthala, IRMC.

Dijkink, G. (1998). Geopolitical codes and popular representations. GeoJournal, 46(4), 292-299.

Doise, W. (1990). Les représentations sociales. In C. Bonnet, R. Ghiglione, T.F. Richard (ed.), Traité de psychologie cognitive, Paris, Dunod.

Gallissot, R. (1987). Sous l'identité, le procès d'identification. L'Homme et la société, 83,12-27.

Gourou, P. (1984). Riz et civilisation. Paris, Fayard.

Grataloup, C. \& Capdepuy, V. (2013). Continents et océans : le pavage européen du globe. Monde(s), 3(1), 29-51.

Gurr, T.R. (1994). Peoples against the State: Ethnopolitical Conflict and the ChangingWorld System. International Studies Quarterly, 38(3), 347-377.

Gurr, T.R. (2000). Peoples Versus States: Minorities at Risk in the New Century. Washington DC, United States Institute of Peace Press.

Henrikson, A.K. (1980). The Geographical "Mental Maps" of American Foreign Policy Makers. International Political Science Review, 1(4), 495-530.

Jisi, W. (1995). Conflit de civilisations : fondement théorique et significations pratiques. Partie 1. Cultures \& Conflits, 19-20, parties 1 et 2 . URL : http://journals.openedition.org/conflits/892 ; DOI : $\underline{10.4000 /}$ conflits.892

Jodelet, D. (1984). Représentations sociales : phénomènes, concept et théories. In Moscovici S. (ed.), Psychologie sociale, Paris, PUF, 361-382.

Jodelet, D. (1997). Représentations sociales : un domaine en expansion. In Jodelet D. (ed.), Les représentations sociales, Paris, PUF, 47-78.

Kolossov, V. (2002). Mir glazami rossiyan: Obchtchesvennoe mneniyei vnechnaya politika. Moscou, Fondation pour l'opinion publique, FOM.

Kolossov, V. (2003). High and Low Geopolitics: Images of Foreign Countries in the Eyes of Russian Citizens. Geopolitics, 8(1), 121-148.

Lacoste, Y. (1993). (ed.), Dictionnaire de géopolitique. Paris, Flammarion. 
Moscovici, S. (1961). La psychanalyse, son image et son public. Paris, PUF.

Mueller, J. (1995). Scénario catastrophe : désordre après la guerre froide. Partie 1. Cultures \& Conflits, 19-20, parties 1 et 2. URL: http://journals.openedition.org/ conflits/878; DOI : $10.4000 /$ conflits.878

Nierop, T. (2001). The clash of civilisations: culture conflict, the state and geographical scale. In Dijking G., Knippenberg H. (eds.), The Territorial Factor. Political Geography in a Globalising World, Amsterdam, Vossiupers UVA, 51-76.

O’Loughlin, J., O’Thuatail, G. \& Kolossov, V. (2005). Russian geopolitical culture and public opinion: the masks of Proteus revisited. Transactions, Institute of British Geographers, 30(3), 322-335.

O’Loughlin, J. \& Talbot, P.F. (2005). Where in the World is Russia? Geopolitical Perceptions and Preferences of Ordinary Russians. Eurasian Geography and Economics, 46(1), 23-50.

O'Tuathail, G., Dalby, S. \& Routledge, P. (1997). The Geopolitics Reader. Routledge, Abingdon-onThames.

Prévélakis, G. (2013). Présentation. Anatoli, 4, 7-11.

Rosière, S. (ed.) (2008). Dictionnaire de l'espace politique. Paris, Armand Colin.

Russett, B., Oneal, J.R. \& Cox, M. (2000). Clash of Civilizations, or Realism and Liberalism Deja Vu? Some Evidence. Journal of Peace Research, 37(5), 583-608.

Saarinen, T.F. (1998). Centering of mental maps of the world. National geographic research, 4, 112-127.

Spengler, O. (2000). Le déclin de l'Occident. Paris, Gallimard, première édition française en 1948. Édition originale Der Untergang des Abendlandes. Umrisse einer Morphologie der Welt-Geschichte, Wien, Leipzig, Wilhelm Braumüller, 1918-1922.

Taillard, C. (ed.) (2004). Intégrations régionales en Asie orientale. Paris, Les Indes savantes.

Toynbee, A. (1934-1961). A Study of History. Oxford,Oxford University Press, 12 volumes.

Coordonnées des auteurs :

Clarisse DIDELON LOISEAU

Professeur des universités

Université Panthéon-Sorbonne

UMR Géographie-cités - CNRS clarisse.didelon-loiseau@univ-paris1.fr

Yann RICHARD

Professeur des universités

Université Panthéon-Sorbonne

UMR PRODIG - CNRS

yrichard@univ-paris1.fr 